\title{
An Observational Study of the Diagnosis and Management of Chronic Urticaria in the UK
}

\author{
Cheng-Han Wu ${ }^{a, d} \quad$ Michael Roger Ardern-Jones ${ }^{a} \quad$ Efrem Eren $^{a, b} \quad$ Carina Venter $^{a, c}$ \\ ${ }^{a}$ MSc Allergy, Faculty of Medicine, University of Southampton, and ${ }^{\text {b }}$ Southampton General Hospital, Southampton, \\ and ' University of Portsmouth, Portsmouth, UK; ${ }^{\mathrm{d}}$ Dalin Tzu Chi Hospital, Buddhist Tzu Chi Medical Foundation, Chiayi, \\ Taiwan, ROC
}

\section{Key Words}

Chronic urticaria · Diagnosis · Guidelines

\begin{abstract}
Background: Patients with chronic urticaria (CU) in the UK could be referred to health care professionals (HCPs) with diverse specialties using different guidelines. The aims of the present study were to determine which CU guidelines HCPs in the UK use, which tests they use for the diagnosis of CU, and how they manage CU. Methods: In this UK-wide survey, we designed a questionnaire covering the diagnosis and management of CU based on current guidelines. The link to the questionnaire was sent to the British Society for Allergy and Clinical Immunology (BSACI), the British Association of Dermatologists (BAD), the British Society of Immunology (BSI), and the Food Allergy and Intolerance Specialist Group (FAISG) of the British Dietetic Association (BDA), who distributed the link to their members. Results: The questionnaire was completed by 55 allergists/immunologists, 64 dermatologists, and 43 dietitians. More dermatologists used the BAD guidelines in comparison with allergists and immunologists (93.6 vs. $12.5 \% ; \mathrm{p}<0.001$ ). On the other hand, the $\mathrm{BSACl}$ guidelines (83.3 vs. $14.9 \% ; \mathrm{p}<0.001$ ) and the European Academy of Allergy and Clinical Immunology (EAACI)/ Global Allergy and Asthma European Network (GA²LEN)/Eu-
\end{abstract}

ropean Dermatology Forum (EDF)/World Allergy Organization (WAO) guidelines (2013) (52.1 vs. 10.6\%; $p<0.001$ ) were used by more allergists and immunologists compared to dermatologists. Differences were found between allergists/ immunologists and dermatologists with regard to guidelines used, investigations performed, preference of first-line antihistamine, and prescription of alternative treatment methods. Conclusion: In conclusion, differences in the diagnosis and management of CU between HCPs of diverse specialties were identified, which reflected differences among the guidelines used.

(c) 2015 S. Karger AG, Basel

\section{Introduction}

Chronic urticaria (CU) is a common disease affecting $0.5-5 \%$ of the general population [1]. Although its exact incidence is uncertain, an estimated incidence of $1 \%$ is generally adopted in the literature [2]. Although CU is not life-threatening, it can reduce the quality of life (QoL) of patients and their performance at work or study significantly. The impact of CU on the QoL of patients has been reported to be comparable with the impact of severe coronary artery disease [3]. Furthermore, while CU is a self-limited disease with a mean duration of 2-5 years,

\section{KARGER 125}

(c) 2015 S. Karger AG, Base

$1018-2438 / 15 / 1671-0001 \$ 39.50 / 0$

E-Mail karger@karger.com

www.karger.com/iaa
Correspondence to: Dr. Carina Venter

School of Health Sciences and Social Work, University of Portsmouth

2 King Richard 1st Road

Portsmouth PO1 2FR (UK)

E-Mail Carina.venter@port.ac.uk 
$20 \%$ of patients suffer from long-lasting CU [2], which may last for more than 20 years [4].

Patients with CU in the UK have the potential to be referred to a range of health care professionals (HCPs). Due to the range of specialist knowledge and available guidelines, it is not surprising that HCPs manage CU patients differently. Differences in laboratory tests requested and medications prescribed for CU between dermatologists, pediatricians, and general practitioners (GPs) were reported in a German survey [5]. Differences in the management of CU between allergists and dermatologists in Spain were reported in the study of Ferrer et al. [6] as well.

The published guidelines on CU released by the European Academy of Allergy and Clinical Immunology (EAACI), the Global Allergy and Asthma European Network $\left(\mathrm{GA}^{2} \mathrm{LEN}\right)$, the European Dermatology Forum (EDF), and the World Allergy Organization (WAO) [79] aim to standardize the management of CU and minimize the differences in management between different HCPs. The German survey reported that physicians familiar with the EAACI guidelines were more likely to prescribe high-dose nonsedating antihistamines and avoid sedating antihistamines and systemic corticosteroids in the management of $\mathrm{CU}$ [5]. In that survey, a greater awareness of the EAACI/GA ${ }^{2} \mathrm{LEN} / \mathrm{EDF} / \mathrm{WAO}$ guidelines was reported in dermatologists compared to pediatricians and GPs. The influence of guidelines could be more complex in the UK because the British Society for Allergy and Clinical Immunology (BSACI) [10] and the British Association of Dermatologists (BAD) [11] have both prepared guidelines. Furthermore, the different opinions from US experts in the latest guidelines developed by the American Academy of Allergy, Asthma, and Immunology (AAAAI), the American College of Allergy, Asthma, and Immunology (ACAAI), and the Joint Council of Allergy, Asthma, and Immunology would create more diversity in the management of CU [1].

Even though the different guidelines for CU have a number of recommendations in common, different practices are still observed. For example, all of the guidelines recommend differential blood count and erythrocyte sedimentation rate (ESR) analyses for the investigation of CU. However, while the EAACI/GA ${ }^{2} \mathrm{LEN} / \mathrm{EDF} / \mathrm{WAO}$ guidelines and the US parameters regard them as routine diagnostic tests, they are only suggested for those who are resistant to antihistamines in the $\mathrm{BAD}$ guidelines. Otherwise, extended diagnostic measures for difficult cases vary with different guidelines. Furthermore, despite the general consensus that second-generation antihistamines are the first-line approach for the management of $\mathrm{CU}$, different alternative treatments for antihistamine-resistant patients have been recommended in individual guidelines. Leukotriene antagonists and ciclosporin have been recommended in all of the guidelines, but in different order of preference. Moreover, there is a discrepancy in the role of sedating antihistamines and systemic corticosteroids in $\mathrm{CU}$ management in different guidelines. Taken together, physicians using different guidelines could manage patients with CU very differently.

All three European guidelines have given recommendations regarding an elimination diet in the management of CU. The evidence of dietary management in CU mainly comes from studies addressing low-pseudoallergen diets $[12,13]$. Pseudoallergens include food additives and some natural food ingredients, such as salicylates and aromatic compounds. On the other hand, some physicians and dietitians might prescribe low-salicylate diets, lowhistamine diets, or low-sulfite diets rather than low-pseudoallergen diets because of the lack of a clear definition of pseudoallergens. Despite the increasing number of studies addressing the efficacy of dietary measures for $\mathrm{CU}$, the popularity of dietary measures in reality is unclear.

The objectives of the present study were to determine which CU guidelines HCPs in the UK use, which tests they use for the diagnosis of $\mathrm{CU}$, and how they manage or treat $\mathrm{CU}$ using an UK-wide questionnaire survey.

\section{Material and Methods}

\section{Study Design and Participants}

This UK-wide cross-sectional survey was conducted in the summer of 2014. HCPs with experience in CU patient care from different regions of the UK were asked to answer online questionnaires via e-mail. The link to the questionnaire was sent to the BSACI, the BAD, the British Society of Immunology (BSI), and the Food Allergy and Intolerance Specialist Group (FAISG) of the British Dietetic Association (BDA), who distributed the link to their members. In addition, it was sent to the South of England Allergy and Immunology Network group. This survey focused on comparisons between allergists, immunologists, dermatologists, and dietitians in the UK; therefore, questionnaires answered by GPs were excluded from the analysis. Questionnaires answered by pediatricians were also excluded because diagnosis and management are different in pediatric CU.

A questionnaire was designed after a literature review and then revised according to comments from an allergist/immunologist, a dermatologist, and a dietitian on the research team. The questionnaires were piloted before being sent to participants. Ten multiplechoice and true-false questions in the questionnaire addressed the following 5 main areas: (a) do HCPs in the UK use CU guidelines, (b) which CU guidelines do they follow, (c) which investigation and management strategies based on current $\mathrm{CU}$ guidelines do 
they use in real life, (d) do they include dietary measures in the management of $\mathrm{CU}$, and (e) do they consider nutrients, including vitamin $D$, vitamin $B_{12}$, and iron in their diagnostic workup? The questionnaire for dietitians had fewer questions, as they neither diagnose nor prescribe medication. To facilitate anonymity, Survey Monkey was used as the online survey tool.

This study was conducted in Southampton General Hospital and was approved by the Faculty of Medicine Ethics Committee of the University of Southampton (ethics ID: 10907).

\section{Statistical and Data Analysis}

For analysis, participants were divided into 3 groups: allergists/ immunologists, dermatologists, and dietitians. Comparisons were made between 2 physicians groups and between dietitians and total physicians. SPSS 21.0 was used for statistical analyses. Comparisons between different groups in the questionnaire survey were tested using Pearson's $\chi^{2}$ test and Fisher's exact test. $\mathrm{p} \leq 0.05$ was considered statistically significant.

\section{Results}

\section{Subjects}

In total, 180 HCPs in the UK completed the questionnaires, including 55 allergists/immunologists, 64 dermatologists, and 43 dietitians. The majority of dietitians were pediatric dietitians (69.8\%). We excluded 3 GPs and 7 pediatricians. Apart from immunology and allergy, responses suggesting multiple specialties were excluded $(\mathrm{n}=8)$ due to the difficulty of placing them in a particular group.

\section{CU Guidelines Used}

In this survey, the use of guidelines for the diagnosis and management of $\mathrm{CU}$ was more frequently observed in allergists and immunologists in comparison to dermatologists ( 90.9 vs. $75.0 \% ; \mathrm{p}=0.023$ ) (table 1 ). Compared to physicians, dietitians used CU guidelines much less frequently ( 81.0 vs. $13.6 \%$; $p<0.001)$. Moreover, $64.9 \%$ of guideline users used only one guideline, while $35.1 \%$ of them used more than one. In total among participating physicians, the BAD guidelines and the BSACI guidelines were the most commonly used (42.3 and $41.5 \%$, respectively), followed by the 2013 revision of the EAACI/ $\mathrm{GA}^{2} \mathrm{LEN} / \mathrm{EDF} / \mathrm{WAO}$ guideline (30.3\%). In contrast, the 2008 version of the EAACI/GA ${ }^{2} \mathrm{LEN} / \mathrm{EDF} / \mathrm{WAO}$ guideline and the US guidelines were rarely used (6.1 and 7.1\%, respectively). Not surprisingly, dermatologists were more likely to use the BAD guidelines in comparison with allergists and immunologists (93.6 vs. $12.5 \%$; $\mathrm{p}<0.001$ ). On the other hand, the BSACI guidelines (83.3 vs. $14.9 \%$; $\mathrm{p}<$ 0.001 ) and the EAACI/GA ${ }^{2} \mathrm{LEN} / \mathrm{EDF} / \mathrm{WAO}$ guideline (2013) (52.1 vs. $10.6 \%$; $<<0.001)$ were more likely to be
Table 1. CU guidelines used by HCPs

\begin{tabular}{lclr}
\hline & $\begin{array}{l}\text { Allergists/ } \\
\text { immunologists } \\
(\mathrm{n}=55)\end{array}$ & $\begin{array}{l}\text { Dermatologists } \\
(\mathrm{n}=64)\end{array}$ & $\begin{array}{l}\mathrm{p} \\
\text { value }\end{array}$ \\
\hline CU guidelines $^{\mathrm{a}}$ & $50(90.9)$ & $48(75.0)$ & 0.023 \\
BAD $^{\mathrm{b}}$ & $6(12.5)$ & $44(93.6)$ & $<0.001$ \\
BSACI $^{\mathrm{b}}$ & $40(83.3)$ & $7(14.9)$ & $<0.001$ \\
${\text { EAACI }(2008)^{\mathrm{b}}}^{\mathrm{b}}$ & $4(8.3)$ & $2(4.3)$ & 0.677 \\
${\text { EAACI }(2013)^{\mathrm{b}}}^{\mathrm{U}}$ & $25(52.1)$ & $5(10.6)$ & $<0.001$ \\
& $6(12.8)$ & $1(2.1)$ & 0.111 \\
\hline
\end{tabular}

Values are presented as numbers (\%) unless otherwise stated. a Some participants used more than one guideline. ${ }^{b}$ Proportion of total guideline users.

used by allergists and immunologists compared to dermatologists.

\section{Investigation}

Allergists and immunologists were more likely to recommend C-reactive protein (CRP) testing (44.7 vs. 10.2\%; $\mathrm{p}<0.001)$, while dermatologists used the ESR more frequently (42.9 vs. $29.8 \%$; $\mathrm{p}=0.184)$ (table 2). Compared to dermatologists, skin prick testing (SPT) was more regularly performed by allergists and immunologists ( $2.0 \mathrm{vs.}$ $44.7 \% ; \mathrm{p}<0.001)$. No statistically significant differences were found between both groups with regard to full blood counts (FBC), antinuclear antibody, thyroid function, thyroid-stimulating hormone (TSH), complement, total immunoglobulin $\mathrm{E}$ (IgE), and specific $\operatorname{IgE}(\mathrm{s} \operatorname{IgE})$. The most common tests used in this survey were FBC (75.9\%), thyroid function (49.1\%), and TSH (50.9\%). In contrast, autologous serum skin tests (ASST) and Helicobacter pylori testing were regularly used by only 2.1 and $9.8 \%$ of the participating physicians, respectively.

Even though the proportion of physicians investigating infection-driven causes for CU was similar in both groups, examinations for dental problems were done more frequently by allergists and immunologists in comparison with dermatologists ( 54.5 vs. $18.4 \% ; \mathrm{p}=0.001)$.

\section{Drugs Prescribed}

All physicians recommended second-generation antihistamines as first-line treatment. Allergists and immunologists tended to prefer cetirizine in comparison to dermatologists ( 62.0 vs. $40.0 \%$; $=0.022$ ), who tend to favor fexofenadine ( 45.0 vs. $24.0 \% ; \mathrm{p}=0.022$ ) (table 3 ). The proportion of physicians using addition of another second-generation antihistamine was similar in both groups 
Table 2. CU investigation performed by HCPs

\begin{tabular}{|c|c|c|c|c|c|}
\hline FBC & $36(76.6)$ & $26(74.3)$ & $36(73.5)$ & $30(83.3)$ & 0.724 \\
\hline CRP & $21(44.7)$ & $15(42.9)$ & $5(10.2)$ & $4(11.1)$ & $<0.001$ \\
\hline ESR & $14(29.8)$ & $10(28.6)$ & $21(42.9)$ & $18(50.0)$ & 0.184 \\
\hline TSH & $24(51.1)$ & $16(45.7)$ & $24(49.0)$ & $20(55.6)$ & 0.838 \\
\hline Complement & $22(46.8)$ & $17(48.6)$ & $16(32.7)$ & $12(33.3)$ & 0.156 \\
\hline $\operatorname{IgE}$ & $8(17.0)$ & $5(14.3)$ & $6(12.2)$ & $5(13.9)$ & 0.507 \\
\hline sIgE & $4(8.5)$ & $2(5.7)$ & $5(10.2)$ & $3(8.3)$ & 1.000 \\
\hline SPT & $21(44.7)$ & $16(45.7)$ & $1(2.0)$ & $1(2.8)$ & $<0.001$ \\
\hline ASST & $3(6.4)$ & $1(2.9)$ & 0 & 0 & 0.113 \\
\hline
\end{tabular}

Values are presented as numbers (\%) unless otherwise stated. ANA = Antinuclear antibody.

${ }^{a}$ All allergists/immunologists vs. all dermatologists. ${ }^{b}$ Including a clinical history, oral examination (gingivitis, tooth abscess, and other dental problems), sinus examination, nail examination (chronic fungal infection), H. pylori testing, parasitic infection (stool sample for ova, cysts, and parasites), and other examinations specified by the respondents.

(allergists/immunologists: $70.0 \%$ vs. dermatologists: 75.8\%). Among additional second-generation antihistamine users, a similar proportion of second-generation antihistamine combination users was found in both groups (allergists/immunologists: $37.1 \%$ vs. dermatologists: $42.6 \%)$. Compared to allergists and immunologists, dermatologists were more likely to consider additional second-generation antihistamines before updosing the original one ( 17.0 vs. $5.9 \%$; $\mathrm{p}=0.180$ ).

The frequency of alternative treatments prescribed was evaluated based on prescriptions in the past 2 months (table 3). Montelukast and $\mathrm{H}_{2}$ antagonists were the leading alternative treatments in this survey (60.2 and 56.5\%, respectively), followed by first-generation antihistamines (50.9\%). Compared to dermatologists, allergists and immunologists were more likely to prefer montelukast as the alternative treatment when second-generation antihistamines failed (61.7 vs. $25.0 \% ; \mathrm{p}<0.001)$. In contrast, $\mathrm{H}_{2}$ antagonists were more frequently chosen by dermatologists as the favored alternative treatment in comparison with allergists and immunologists (43.3 vs. $17.0 \%$; $\mathrm{p}=0.004$ ). However, physicians in both groups (allergists/immunologists: $63.2 \%$ vs. dermatologists: $71.7 \%$ ) stated that montelukast was only effective in less than $25 \%$ of patients. On the other hand, ciclosporin (54.1 vs. $29.8 \% ; \mathrm{p}=0.012$ ) and cooling antipruritic lotion (45.9 vs.
$25.5 \% ; \mathrm{p}=0.030$ ) were prescribed by more dermatologists in the past 2 months compared to allergists and immunologists. No differences were found with regard to the frequency of prescription of $\mathrm{H}_{2}$ blockers, first-generation antihistamines, regularly but not short-term used oral corticosteroid, tricyclic antidepressants, dapsone, or omalizumab in both groups.

\section{Dietary Measures}

Dietary measures were used by only $25.4 \%$ of the participating physicians. Among the dietary measures used, in both physicians and dietitians, the most commonly used elimination diets were low-salicylate diets (physicians: $72.4 \%$ vs. dietitians: $60.0 \%$ ), while low-pseudoallergen diets were much less frequently used (physicians: $20.7 \%$ vs. dietitians: $13.3 \%$ ) (table 4 ).

Among the nutrients surveyed, iron was the most frequently investigated by the participating physicians (10.4\%), while vitamin D was considered important by most participating dietitians (23.7\%). Compared to dermatologists, allergists and immunologists checked vitamin D levels more frequently (10.6 vs. $0.0 \% ; \mathrm{p}=0.025$ ). Likewise, vitamin D supplementation was undertaken by allergists and immunologists more frequently compared to dermatologists (38.5 vs. $0.0 \% ; \mathrm{p}=0.016)($ table 5$)$. 
Table 3. Drugs prescribed by HCPs in the management of CU

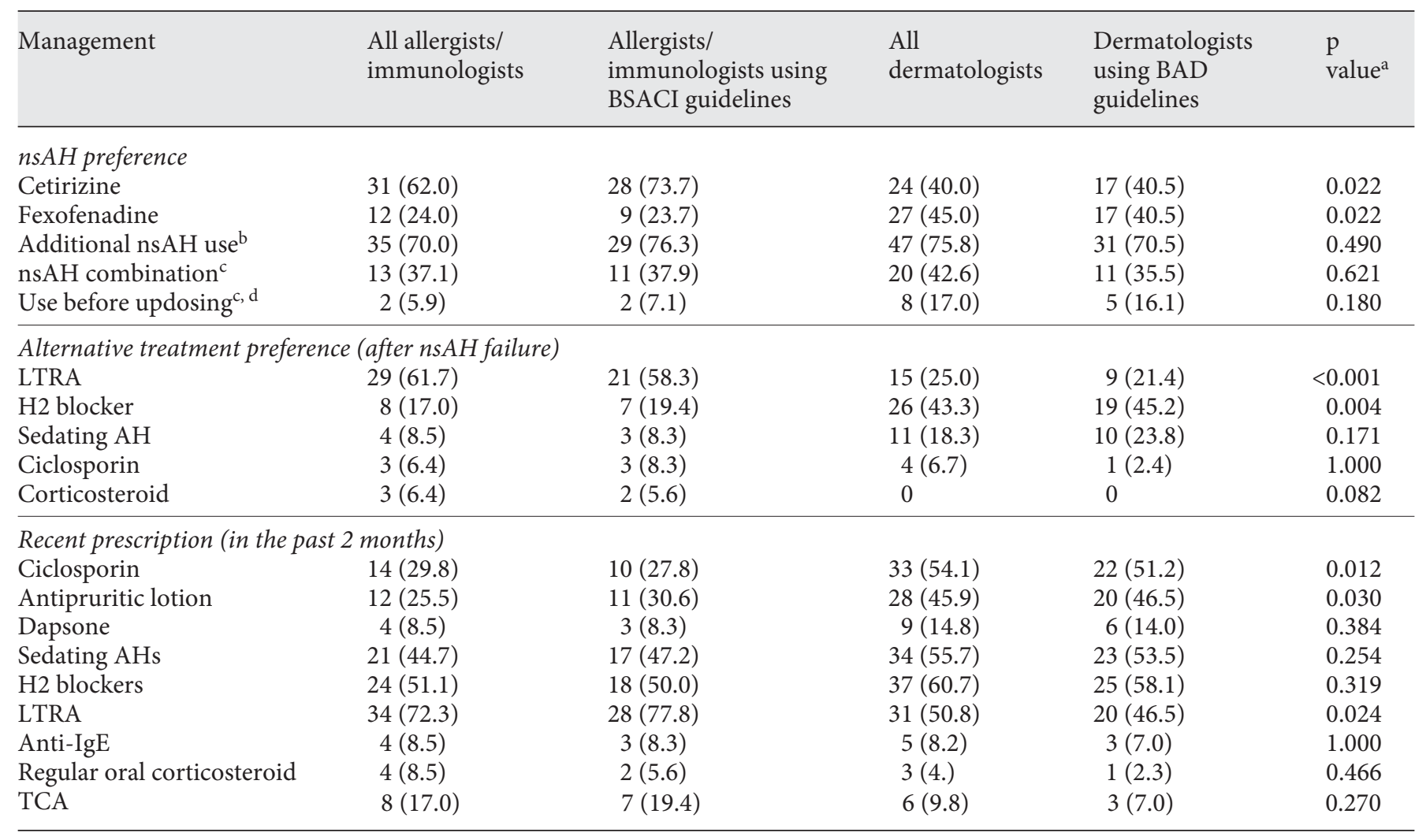

Values are presented as numbers (\%) unless otherwise stated. nsAH = Nonsedating antihistamine; LTRA = leukotriene receptor antagonist; TCA = tricyclic antidepressants. ${ }^{\mathrm{a}}$ All allergists/immunologists vs. all dermatologists. ${ }^{\mathrm{b}}$ Prescription of a different nsAH when the first-line nsAH has failed. ${ }^{c}$ Proportion of total additional nsAH users. ${ }^{\mathrm{d}}$ Prescription of additional nsAH before updosing treatment with the first-line nsAH.

\section{Discussion}

In this study we set out to find out if different groups of HCPs diagnose and manage $\mathrm{CU}$ differently and if that is related to the guidelines they use. We sent out a questionnaire to BSACI, BAD, and FAISG members. We found that different groups of HCPs tend to deal with $\mathrm{CU}$ in different ways. Differences in guidelines used were also observed, which may be part of the causation of the different practices. In our survey, dietary measures and nutrient deficiencies were seldom considered in the management of CU.

This is the first study to address the differences in CU guidelines used among HCPs. While the mainstream guidelines used by the participating UK physicians in the 2 groups were the $\mathrm{BSACI}$ guidelines and the $\mathrm{BAD}$ guidelines, respectively, there were fewer participants using the EAACI/GA ${ }^{2} \mathrm{LEN} / \mathrm{EDF} / \mathrm{WAO}$ guidelines and the US
Table 4. Users of CU guidelines and dietary measures among physicians and dietitians

\begin{tabular}{lccc}
\hline & $\begin{array}{l}\text { Physicians } \\
(\mathrm{n}=125)\end{array}$ & $\begin{array}{l}\text { Dietitians } \\
(\mathrm{n}=42)\end{array}$ & $\begin{array}{l}\mathrm{p} \\
\text { value }\end{array}$ \\
\hline CU guideline users & $102 / 126(81.0 \%)$ & $6 / 44(13.6 \%)$ & $<0.001$ \\
$\begin{array}{c}\text { Dietary measure users } \\
\text { Low-salicylate diet }\end{array}$ & $\begin{array}{l}29 / 114(25.4 \%) \\
1 / 29(72.4 \%)\end{array}$ & $\begin{array}{c}18 / 34(52.9 \%) \\
9 / 15(60.0 \%)\end{array}$ & 0.003 \\
$\begin{array}{l}\text { Pseudoallergen-free } \\
\text { diet }\end{array}$ & $6 / 29(20.7 \%)$ & $2 / 15(13.3 \%)$ & 0.695 \\
$\begin{array}{l}\text { Low-histamine diet } \\
\text { Low-sulfite diet }\end{array}$ & $8 / 29(27.6 \%)$ & $8 / 15(53.3 \%)$ & 0.092 \\
$\begin{array}{l}\text { Vitamin D supple- } \\
\text { mentation }\end{array}$ & $4 / 29(13.8 \%)$ & $4 / 15(26.7 \%)$ & 0.414 \\
& & & \\
& $5 / 29(17.2 \%)$ & $7 / 15(46.7 \%)$ & 0.038 \\
\hline
\end{tabular}


Table 5. Dietary measures used and micronutrients surveyed by HCPs in the management of CU

\begin{tabular}{|c|c|c|c|c|c|}
\hline & $\begin{array}{l}\text { All allergists/ } \\
\text { immunologists }\end{array}$ & $\begin{array}{l}\text { Allergists/ } \\
\text { immunologists using } \\
\text { BSACI guidelines }\end{array}$ & $\begin{array}{l}\text { All } \\
\text { dermatologists }\end{array}$ & $\begin{array}{l}\text { Dermatologists } \\
\text { using BAD } \\
\text { guidelines }\end{array}$ & $\begin{array}{l}\mathrm{p} \\
\text { value }^{\mathrm{a}}\end{array}$ \\
\hline \multicolumn{6}{|l|}{ Micronutrient investigation } \\
\hline Vitamin D & $5(10.6)$ & $4(11.4)$ & 0 & 0 & 0.025 \\
\hline Vitamin $\mathrm{B}_{12}$ & $1(2.1)$ & $1(2.9)$ & 0 & 0 & 0.490 \\
\hline Ferritin & $7(14.9)$ & $4(11.4)$ & $3(6.1)$ & $1(2.8)$ & 0.194 \\
\hline Low-salicylate diets ${ }^{\mathrm{b}}$ & $10(76.9)$ & $10(90.9)$ & $9(64.3)$ & $7(58.3)$ & 0.678 \\
\hline Pseudoallergen-free diets ${ }^{b}$ & $3(23.1)$ & $2(18.2)$ & $2(14.3)$ & $2(16.7)$ & 0.648 \\
\hline Low-histamine diets ${ }^{\mathrm{b}}$ & $3(23.1)$ & $2(18.2)$ & $4(28.6)$ & $4(33.3)$ & 1.000 \\
\hline Low-sulfite diets ${ }^{\mathrm{b}}$ & $3(23.1)$ & $3(27.3)$ & $1(7.1)$ & $1(8.3)$ & 0.326 \\
\hline Vitamin D supplements ${ }^{\mathrm{b}}$ & $5(38.5)$ & $4(36.4)$ & 0 & 0 & 0.016 \\
\hline
\end{tabular}

Values are presented as numbers (\%) unless otherwise stated. ${ }^{a}$ All allergists/immunologists vs. all dermatologists. ${ }^{b}$ Proportion of the total dietary measure users.

guidelines. Therefore, if the behavior of HCPs can be influenced by the guidelines used, it is reasonable to assume differences in the management of CU not only between HCPs of different specialties but also between the UK, Europe, and the US. Regarding the implementation of CU guidelines, a higher proportion of guideline users was observed in allergists and immunologists in our survey. While $70 \%$ of UK dermatologists used the BAD guidelines in our study, the proportion of German dermatologists reported to follow the EAACI/GA ${ }^{2} \mathrm{LEN} / \mathrm{EDF} / \mathrm{WAO}$ guidelines in the study of Weller et al. [5] was $40 \%$. If the proportion of users of the mainstream guidelines could roughly reflect the proportion of total guidelines users, the implementation of $\mathrm{CU}$ guidelines might be better in the UK.

In terms of tests suggested, FBC and ESR/CRP were recommended in all of the current CU guidelines. While both the BSACI guidelines and the BAD guidelines only recommended ESR $[10,11]$, the EAACI/GA ${ }^{2} \mathrm{LEN} / \mathrm{EDF} /$ WAO guidelines suggested either ESR or CRP [9]. The higher proportion of users of the EAACI/GA ${ }^{2} \mathrm{LEN} / \mathrm{EDF} /$ WAO guidelines in the allergist/immunologist group might be the reason why participants in this group performed CRP tests more frequently. On the other hand, the BSACI guidelines but not the BAD guidelines recommended SPTs to exclude atopic diathesis [10], which could account for the difference in SPT use between both groups in our survey. Because allergy in Germany is linked to, among other things, dermatology, German dermatologists were reported to perform SPT frequently
$(84.5 \%)$ [5]. Instead, it was used regularly in only $2 \%$ of the participating UK dermatologists in our survey.

Taken together, these differences in the investigation of $\mathrm{CU}$ also exist between the UK and Europe. Instead of FBC, total IgE, and SPT, reported as the most commonly performed laboratory tests in the German survey [5], the most common tests reported in our survey were FBC, thyroid function, and TSH. One possible explanation might be that thyroid function was the only test recommended together with FBC and ESR in the BAD guidelines [11], while it was only one of the extended diagnostic measures in the EAACI/GA ${ }^{2} \mathrm{LEN} / \mathrm{EDF} / \mathrm{WAO}$ guidelines [9].

There were some similarities and some discrepancies regarding recommendations for the medical management of CU in the guidelines. Second-generation antihistamines were suggested as the first-line treatment in all of the current CU guidelines. There were more physicians following this recommendation in our survey $(100 \%)$ in comparison with the German survey (dermatologists: nearly 45\%) [5]. The difference might have resulted from the higher proportion of guideline users in the UK and the increasing awareness of nonsedating antihistamine use these days. Moreover, the BSACI guidelines stated that cetirizine might be more effective than other secondgeneration antihistamines [10]. This may be the reason why more allergists and immunologists preferred cetirizine while more dermatologists preferred fexofenadine in this survey. 
While sedating antihistamines were strongly advocated against by the EAACI/GA ${ }^{2} \mathrm{LEN} / \mathrm{EDF} / \mathrm{WAO}$ guidelines [9], they were listed as a reasonable alternative treatment in the BSACI and the BAD guidelines $[10,11]$. This might account for the frequent prescription of first-generation antihistamines in this survey. The stepwise treatment algorithm in the BSACI guidelines recommends montelukast after failure of antihistamines [10], which might play a role in the preference for montelukast in participating allergists and immunologists when secondgeneration antihistamines have failed. In contrast, there was no stepwise treatment algorithm provided in the $\mathrm{BAD}$ guidelines. The recommendation of additional second-generation antihistamine use following updosing treatment of the original one in the same algorithm might be the reason why fewer allergists and immunologists considered additional second-generation antihistamines before antihistamine updosing in our survey [10], although this finding was not statistically significant.

The guidelines also showed differences in terms of their recommendations regarding dietary measures for $\mathrm{CU}$. While low-pseudoallergen diets were recommended in the EAACI/GA ${ }^{2} \mathrm{LEN} / \mathrm{EDF} / \mathrm{WAO}$ guidelines [9], the favored dietary measure in the BSACI guideline was a lowsalicylate diet [10]. This might be the reason why most UK participants prescribed a low-salicylate diet. However, overall, few participants used a low-pseudoallergen diet, which is more mainstream in Europe. In view of the low proportion of dietary measure users, the recommendations regarding dietary management in the current $\mathrm{CU}$ guidelines seem to be unconvincing to most participating clinicians in our study. On the other hand, the low proportion of dietitians seeing adults (30.2\% in this survey) might contribute to the low profile of dietary measures used by participating physicians in the management of CU in the UK.

Even though the role of vitamin D in the etiology and management of CU has been discussed for some time [14-16], most participating clinicians were not convinced. Rorie et al. [14] reported that vitamin D supplements improved CU outcomes regardless of the level of serum vitamin $\mathrm{D}$. However, the dose of vitamin $\mathrm{D}$ given in this study was far more than the usual dose recommended. On the other hand, in this survey, there were more physicians considering iron deficiency in the management of CU compared to vitamin D. To our knowledge, iron status has not been previously studied in CU patients.

Data on the diagnosis and management of $\mathrm{CU}$ in this study was gathered from a questionnaire survey, which is prone to confounding factors. Although we pilot studied the questionnaire, it would be important to validate the findings with actual clinical practice to establish that the answers given truly reflect the physician/dietician behavior. However, despite these limitations, we were reassured that the data supported the expected preference of allergists/immunologists to follow BSACI guidelines in name and practice and of the dermatologists to do likewise with BAD guidelines. Furthermore, the differences highlighted here reflect differences among different guidelines.

Our findings suggest that the differences between $\mathrm{CU}$ guidelines strongly contribute to the different practices between HCPs of different specialties and between the UK and Europe. Considering the possible confusion resulting from so many different guidelines, we suggest an evidence-based unifying guideline, not only for the UK but ideally globally, to harmonize treatment with regard to the best evidence and to provide patients with the same level of care independently of the type of HCP they consult and the country they live in. To achieve this, the different societies need to better cooperate with each other in order to start joint efforts.

\section{Acknowledgements}

We thank all of the participants, the BSACI, the BAD, the BSI, and the FAISG of the BDA. We are also grateful for the support of the University of Southampton (MSc Allergy).

References diagnosis and management of acute and chronic urticaria: 2014 update. J Allergy Clin Immunol 2014;133:1270-1277.

2 Saini SS: Urticaria and angioedema; in Adkinson NF Jr, Burks AW, Busse WW, Holgate ST, Lemanske RF Jr, O'Hehir RE (eds): Middleton's Allergy: Principles and Practice, ed 8. Amsterdam, Elsevier, 2014, pp 575587.

-3 O’Donnell BF, Lawlor F, Simpson J, Morgan M, Greaves MW: The impact of chronic urticaria on the quality of life. Br J Dermatol 1997; 136:197-201.

4 Philpott H, Kette F, Hissaria P, Gillis D, Smith W: Chronic urticaria: the autoimmune paradigm. Intern Med J 2008;38:852857.

5 Weller K, Viehmann K, Brautigam M, et al: Management of chronic spontaneous urticaria in real life - in accordance with the guidelines? A cross-sectional physician-based survey study. J Eur Acad Dermatol Venereol 2013;27:43-50. 
6 Ferrer M, Jauregui I, Bartra J, et al: Chronic urticaria: do urticaria nonexperts implement treatment guidelines? A survey of adherence to published guidelines by nonexperts. $\mathrm{Br}$ J Dermatol 2009;160:823-827.

-7 Zuberbier T, Asero R, Bindslev-Jensen C, et al: EAACI/GA(2)LEN/EDF/WAO guideline: management of urticaria. Allergy 2009;64: 1427-1443.

8 Zuberbier T, Asero R, Bindslev-Jensen C, et al: EAACI/GA(2)LEN/EDF/WAO guideline: definition, classification and diagnosis of urticaria. Allergy 2009;64:1417-1426.

9 Zuberbier T, Aberer W, Asero R, et al: The EAACI/GA(2)LEN/EDF/WAO guideline for the definition, classification, diagnosis, and management of urticaria: the 2013 revision and update. Allergy 2014;69:868-887.
10 Powell RJ, Du Toit GL, Siddique N, et al: BSACI guidelines for the management of chronic urticaria and angio-oedema. Clin Exp Allergy 2007;37:631-650.

11 Grattan CE, Humphreys F; British Association of Dermatologists Therapy Guidelines and Audit Subcommittee: Guidelines for evaluation and management of urticaria in adults and children. Br J Dermatol 2007;157: 1116-1123.

12 Magerl M, Pisarevskaja D, Scheufele R, Zuberbier T, Maurer M: Effects of a pseudoallergen-free diet on chronic spontaneous urticaria: a prospective trial. Allergy 2010;65:78-83.
13 Zuberbier T, Chantraine-Hess S, Hartmann K, Czarnetzki BM: Pseudoallergen-free diet in the treatment of chronic urticaria: a prospective study. Acta Derm Venereol 1995;75:484487

14 Rorie A, Goldner WS, Lyden E, Poole JA: Beneficial role for supplemental vitamin $\mathrm{D}_{3}$ treatment in chronic urticaria: a randomized study. Ann Allergy Asthma Immunol 2014; 112:376-382.

15 Thorp WA, Goldner W, Meza J, Poole JA: Reduced vitamin $D$ levels in adult subjects with chronic urticaria. J Allergy Clin Immunol 2010;126:413; author reply 413-414.

16 Grzanka A, Machura E, Mazur B, et al: Relationship between vitamin $\mathrm{D}$ status and the inflammatory state in patients with chronic spontaneous urticaria. J Inflamm 2014;11:2. 